\title{
Teor de nutrientes e trocas gasosas em plantas de copo-de-leite cultivadas em sistema hidropônico
}

\author{
Paulo Roberto Corrêa Landgraf(1), Douglas José Marques(1), José Ricardo Mantovani(1), \\ Adriano Bortolotti da Silva( ${ }^{(1)}$, Breno Régis Santos ${ }^{(2)}$, Patrícia Duarte de Oliveira Paiva ${ }^{(3)}$ e Rodrigo Sérgio da Silva ${ }^{(1)}$ \\ (1)Universidade José do Rosário Vellano, Rodovia MG 179, Km 0, Campus Universitário, CEP 37130-000 Alfenas, MG, Brasil. E-mail: \\ paulo.landgraf@unifenas.br,douglas.marques@unifenas.br,mantovanij@@yahoo.com,adriano.silva@unifenas.br,rodrigo_silva@agronomo.eng.br \\ (2)Universidade Federal de Alfenas, Instituto de Ciências da Natureza, Rua Gabriel Monteiro da Silva, no 700, Centro, CEP 37130-000 Alfenas, \\ MG, Brasil. E-mail: brenors@yahoo.com.br ${ }^{(3)}$ Universidade Federal de Lavras, Caixa Postal 3037, CEP 37200-000 Lavras, MG, Brasil. E-mail: \\ patriciapaiva@dag.ufla.br
}

Resumo - O objetivo deste trabalho foi avaliar o efeito do cultivo de plantas de copo-de-leite (Zantedeschia aethiopica) em sistema hidropônico, no crescimento vegetativo, no teor de nutrientes na parte aérea e nas trocas gasosas. Utilizou-se o delineamento experimental inteiramente casualizado, em arranjo fatorial triplo ( $2 \times 2 \times 2)$, com oito tratamentos, três repetições e oito plantas por parcela. Os tratamentos consistiram de

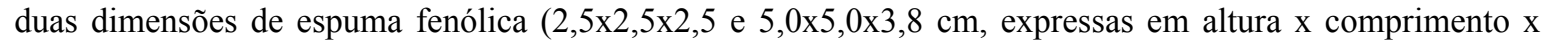
largura), duas soluções nutritivas e dois perfis hidropônicos (100 e $150 \mathrm{~mm}$ ). As plantas de copo-de-leite foram cultivadas em sistema hidropônico fluxo laminar de nutriente (NFT) e apresentaram teores de nutrientes na parte aérea, na seguinte sequência decrescente: $\mathrm{K}>\mathrm{N}>\mathrm{Ca}>\mathrm{P}>\mathrm{S}>\mathrm{Mg}$ para macronutrientes e $\mathrm{Fe}>\mathrm{Mn}>\mathrm{Zn}>\mathrm{B}>\mathrm{Cu}$ para micronutrientes. Plantas de copo-de-leite cultivadas em sistema hidropônico NFT, em espuma fenólica de 5,0x5,0x3,8 cm e em perfil hidropônico de $150 \mathrm{~mm}$, apresentam maior acúmulo de massa de matéria seca na parte aérea e no rizoma, bem como maiores taxas fotossintéticas, independentemente da solução nutritiva.

Termos para indexação: Zantedeschia aethiopica, floricultura, fotossíntese, nutrição mineral.

\section{Nutrient content and gas exchange in calla lily plants grown in a hydroponic system}

\begin{abstract}
The objective of this work was to evaluate the effect of growing calla lily plants (Zantedeschia aethiopica) in a hydroponic system on vegetative growth, nutrient content in shoots, and gas exchange. A completely randomized design in a triple factorial arrangement $(2 \times 2 \times 2)$ was used, with eight treatments, three replicates, and eight plants per plot. The treatments consisted of two dimensions of phenolic foam $(2.5 \times 2.5 \times 2.5$ and $5.0 \times 5.0 \times 3.8 \mathrm{~cm}$, expressed as height $\mathrm{x}$ length $\mathrm{x}$ width), two nutrient solutions, and two hydroponic profiles $(100$ and $150 \mathrm{~mm}$ ). The calla lily plants were grown in a nutrient film technique (NFT) hydroponic system and presented nutrient content in the shoots in the following decreasing order: $\mathrm{K}>\mathrm{N}>\mathrm{Ca}>\mathrm{P}>\mathrm{S}>\mathrm{Mg}$ for macronutrients and $\mathrm{Fe}>\mathrm{Mn}>\mathrm{Zn}>\mathrm{B}>\mathrm{Cu}$ for micronutrients. Calla lily plants grown in a NFT hydroponic system, in the phenolic foam of 5.0x5.0x3.8-cm dimensions and in the hydroponic profile of $150 \mathrm{~mm}$, show greater dry matter accumulation in shoots and bulbs, as well higher photosynthetic rates, regardless of nutrient solution.
\end{abstract}

Index terms: Zantedeschia aethiopica, floriculture, photosynthesis, mineral nutrition.

\section{Introdução}

O número de produtores de flores e plantas ornamentais no País elevou-se de 6.023 para 7.800 , entre 2008 e 2013 , enquanto a área cultivada nesse mesmo período passou de 11.916 para 13.468 ha (Junqueira \& Peetz, 2014). Segundo os autores, entre os fatores que colaboraram para o crescimento do setor, estão o surgimento e o fortalecimento de novos polos produtores, que favorecem a maior oferta regional de produtos, com redirecionamento para o mercado interno, em consequência da situação de retração do mercado externo instaurada desde 2008.

Entre as espécies ornamentais rizomatosas mundialmente comercializadas, destaca-se o copo-de-leite [Zantedeschia aethiopica (L.) Spreng.], muitoapreciado pela versatilidade de uso e beleza das inflorescências. O copo-de-leite é a espécie mais comercializada do seu gênero, e apresenta folhas verdes de aspecto brilhante e flores brancas (Ribeiro et al., 2009). Em Minas Gerais, o seu cultivo está distribuído em várias regiões, com predomínio de produtores (44\%) na região Sul do 
estado, atribuído principalmente ao clima favorável para o desenvolvimento da cultura (Landgraf \& Paiva, 2010).

A produção de copo-de-leite para corte é basicamente conduzida por produtores familiares que utilizam áreas marginais em suas propriedades, como, por exemplo, áreas de várzea. Nessas áreas, o controle de doenças, em especial da podridão-mole, causada pela bactéria de solo Pectobacterium carotovorum, Enterobacteriaceae, é bastante dificultada, o que pode causar perda total da produção (Garcia-Garza et al., 2004). Essa bactéria é o principal agente fitopatogênico da cultura e pode ser disseminada durante o processo de propagação vegetativa, realizado por divisão de touceiras ou de rizomas (Figueiredo et al., 2014). Além disso, no cultivo de copo-de-leite em áreas de várzea, também não é possível ter precisão na adubação, o que pode ocasionar desperdícios e contaminação ambiental pela adubação excessiva ou deficiência nutricional nas plantas pela adubação/nutrição limitada.

Uma das alternativas para evitar a infecção por $P$. carotovorum é o cultivo do copo-de-leite no sistema hidropônico, a partir de mudas com alta qualidade fitossanitária, as quais podem ser obtidas por técnicas de cultura de tecidos de plantas, como a cultura de ápices caulinares (Chang et al., 2003). Nesse sistema, as plantas podem apresentar crescimento mais rápido, o que encurta o ciclo produtivo e aumenta a produtividade e a qualidade pós-colheita (Schmidt et al., 2001).

No Brasil, há carência de informação sobre o cultivo de flores em sistema hidropônico. Recomendações sobre o manejo da nutrição e da adubação para o cultivo de copo-de-leite, com base na extração de nutrientes pelas plantas, também ainda são bastante escassas (Carneiro et al., 2015); isso tem ocasionado o uso inadequado de fertilizantes, os quais têm sido aplicados em quantidades elevadas ou reduzidas, o que compromete o bom desenvolvimento, a produtividade e a qualidade das flores (Silberbush \& Lieth, 2004).

O objetivo deste trabalho foi avaliar o efeito do cultivo de plantas de copo-de-leite (Zantedeschia aethiopica) em sistema hidropônico, no crescimento vegetativo, no teor de nutrientes na parte aérea e nas trocas gasosas.

\section{Material e Métodos}

As plantas de copo-de-leite utilizadas no experimento foram obtidas por técnicas de micropropagação, a partir da cultura in vitro de ápices caulinares. Após 4 meses de cultivo in vitro, as plantas foram uniformizadas com, aproximadamente, dois pares de folhas, antes da sua transferência para o cultivo hidropônico em sistema fluxo laminar de nutriente (NFT).

O experimento foi conduzido no Setor de Olericultura e Experimentação da Universidade José do Rosário Vellano, em Alfenas, MG, no período de fevereiro de 2013 a dezembro de 2014.

Utilizou-se o delineamento experimental inteiramente casualizado, constituído de arranjo fatorial triplo ( $2 \times 2 \times 2)$, com duas dimensões de espuma fenólica $(2,5 \times 2,5 \times 2,5$ e $5,0 \times 5,0 \times 3,8 \mathrm{~cm}$, expressas em altura $\mathrm{x}$ comprimento $\mathrm{x}$ largura), dois perfis hidropônicos (100 e $150 \mathrm{~mm}$ ) e duas soluções nutritivas, o que totalizou oito tratamentos, com três repetições e oito plantas por parcela.

As plantas foram mantidas em casa de vegetação modelo arco, com $126 \mathrm{~m}^{2}$ (6,0 m de largura por $21 \mathrm{~m}$ de comprimento) e pé direito de $3,0 \mathrm{~m}$. A estrutura foi coberta com película de polietileno leitoso (150 microns), além de tela de sombreamento de $80 \%$ na face superior e nas laterais. Foram utilizadas duas bancadas: uma para a solução hidropônica A e a outra para a solução B. Cada bancada apresentou $1 \%$ de declividade e tinha $9,0 \mathrm{~m}$ de comprimento com 12 canais de cultivo, em que os tratamentos espuma fenólica e perfil hidropônico foram dispostos.

As concentrações de nutrientes das soluções nutritivas A e B (Tabela 1) foram derivadas de acordo com Postali et al. (2004) e ajustadas para o experimento. Nas soluções nutritivas, foram utilizados os seguintes fertilizantes: nitrato de potássio $(36 \%$ de $\mathrm{K}$ e $\left.13 \% \mathrm{~N}_{-} \mathrm{NO}_{3}^{-},\right)$, nitrato de cálcio $(17 \%$ de Ca e $12 \%$ de $\mathrm{N}^{-} \mathrm{NO}_{3}{ }^{-}$), sulfato de magnésio ( $10 \%$ de $\mathrm{Mg}$ e $13 \%$ de S), fosfato monobásico de potássio (29\% de K e $23 \%$ de $\mathrm{P})$, ácido bórico ( $17 \%$ de B), sulfato de cobre $(24 \%$ de $\mathrm{Cu}$ e $12 \%$ de $\mathrm{S}$ ), sulfato de manganês $(25 \%$ de $\mathrm{Mn}$ e $21 \%$ de S), sulfato de zinco ( $22 \%$ de $\mathrm{Zn}$ e $11 \%$ de S), molibdato de sódio (39\% de Mo) e ferrilene $(6 \%$ de $\mathrm{Fe}$ ).

Cada solução nutritiva foi mantida em reservatório de fibra de amianto com capacidade de 250 L. As irrigações foram realizadas a cada $15 \mathrm{~min} \mathrm{e}$ controladas automaticamente por temporizador. No período noturno, o temporizador foi programado para fazer três irrigações: às $20 \mathrm{~h} 00,01 \mathrm{~h} 00$ e $04 \mathrm{~h} 00$. O manejo da solução hidropônica foi realizado 
diariamente, e o volume da solução foi completado até o inicial. No início da manhã, realizou-se medição e ajuste da condutividade elétrica para $2,0-3,0 \mathrm{mS}$ e do $\mathrm{pH}$ para 5,8, nas duas soluções. No transplantio, foi realizado um corte no sistema radicular das mudas para homogeneização, as quais, em seguida, foram transferidas para as bancadas do sistema hidropônico.

Os parâmetros avaliados, taxa de fotossíntese foliar e taxa de transpiração, foram medidos com um sistema medidor portátil de fotossíntese modelo LI-6400XT (Li-Cor, Lincoln, NE, EUA), aos 56 dias do transplantio. As medidas foram realizadas no período da manhã, entre $9 \mathrm{~h} 00$ e $11 \mathrm{~h} 00$, em folha totalmente expandida, e as leituras, no terço mediano das plantas. As medidas foram realizadas em uma área foliar de $6,0 \mathrm{~cm}^{2}$, com fluxo de ar na câmara de $380 \mu \mathrm{mol} \mathrm{mol}{ }^{-1} \mathrm{de}^{\mathrm{CO}_{2}}$. O ar foi coletado de fora do cultivo protegido, transportado para dentro de um galão de proteção e bombeado para a câmara. Foi utilizada uma densidade de fluxo de fótons de $1.500 \mu \mathrm{mol} \mathrm{m} \mathrm{m}^{-2} \mathrm{~s}^{-1}$ de uma fonte de luz red-blue $L E D$, e a temperatura foliar foi de $30^{\circ} \mathrm{C}$.

Após 90 dias de cultivo, foram realizadas avaliações de crescimento das plantas por meio da massa de matéria seca da parte aérea e do rizoma. Os teores dos nutrientes na parte aérea (caule+folhas) foram determinados de acordo com Malavolta et al. (1997).

Os resultados foram submetidos à análise de variância, e as médias foram comparadas pelo teste de Scott-Knott, por meio do programa Sisvar (Ufla, Lavras, MG).

Tabela 1. Soluções nutritivas utilizadas para o cultivo hidropônico de copo-de-leite (Zantedeschia aethiopica).

\begin{tabular}{lcc}
\hline Nutriente & Solução A & Solução B \\
\hline${\mathrm{N}-\mathrm{NO}_{3}{ }^{-}}^{-}$ & 167 & 236 \\
$\mathrm{P}$ & 64 & 62 \\
$\mathrm{~K}$ & 316 & 430 \\
$\mathrm{Ca}$ & 153 & 161 \\
$\mathrm{Mg}$ & 43 & 24 \\
$\mathrm{~S}$ & 50 & 32 \\
$\mathrm{~B}$ & 0,2 & 0,3 \\
$\mathrm{Cu}$ & 0,03 & 0,05 \\
$\mathrm{Fe}$ & 4,3 & 5,0 \\
$\mathrm{Mn}$ & 1,1 & 0,4 \\
$\mathrm{Mo}$ & 0,05 & 0,05 \\
$\mathrm{Zn}$ & 0,3 & 0,5 \\
\hline
\end{tabular}

\section{Resultados e Discussão}

O perfil hidropônico e o tamanho de espuma fenólica afetaram significativamente as produções de massa de matéria seca do rizoma e da parte aérea (folhas + caule) das plantas de copo-de-leite (Tabela 2). Também foi constatada interação significativa entre esses tratamentos quanto aos parâmetros de desenvolvimento vegetativo das plantas. Além disso, as plantas apresentaram desenvolvimento semelhante nas duas soluções nutritivas empregadas, o que indica que ambas forneceram os nutrientes necessários para $o$ copo-de-leite.

Verificou-se que o uso de espuma fenólica de maior dimensão (EF2, 5,0x5,0x3,8 cm) foi o mais adequado para o desenvolvimento das plantas de copo-de-leite em sistema hidropônico. Isso porque tanto a massa de matéria seca do rizoma quanto a da parte aérea foram cerca de 1,4 vez maior do que a das plantas do tratamento em que foi utilizada a menor espuma

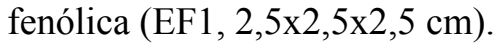

$\mathrm{O}$ perfil hidropônico de $150 \mathrm{~mm}$ (P150 mm) proporcionou rizomas de copo-de-leite aproximadamente 2,5 vezes maiores do que os do perfil de $100 \mathrm{~mm}$ $(\mathrm{P} 100 \mathrm{~mm})$. Contudo, em relação à parte aérea das plantas, o desenvolvimento foi semelhante nos dois perfis hidropônicos utilizados (Tabela 2). O uso de perfil hidropônico e de espuma fenólica de maiores dimensões $(\mathrm{EF} 2 / \mathrm{P} 150 \mathrm{~mm})$, provavelmente, favoreceu o maior

Tabela 2. Massa de matéria seca (MS) do rizoma e da parte aérea (caule+folhas) de copo-de-leite (Zantedeschia aethiopica) em cultivo hidropônico, com diferentes soluções nutritivas, perfis hidropônicos (100 e $150 \mathrm{~mm}$ ) e tamanhos de espuma fenólica $(\mathrm{EF})^{(1)}$.

\begin{tabular}{|c|c|c|c|c|}
\hline \multirow{2}{*}{$\begin{array}{l}\text { Espuma } \\
\text { fenólica }\end{array}$} & \multicolumn{2}{|c|}{ Massa de MS do rizoma (g) } & \multicolumn{2}{|c|}{ Massa de MS da parte aérea $(\mathrm{g})$} \\
\hline & $100 \mathrm{~mm}$ & $150 \mathrm{~mm}$ & $100 \mathrm{~mm}$ & $150 \mathrm{~mm}$ \\
\hline & \multicolumn{4}{|c|}{ Solução A } \\
\hline EF1 & $0,74 \mathrm{Ab}$ & $1,56 \mathrm{Ba}$ & $1,65 \mathrm{Ba}$ & $1,51 \mathrm{Ba}$ \\
\hline \multirow[t]{2}{*}{ EF2 } & $0,53 \mathrm{Ab}$ & $2,12 \mathrm{Aa}$ & $2,08 \mathrm{Ab}$ & 2,42Aa \\
\hline & \multicolumn{4}{|c|}{ Solução B } \\
\hline EF1 & $0,48 \mathrm{Bb}$ & $1,54 \mathrm{Ba}$ & $1,92 \mathrm{Ba}$ & $1,74 \mathrm{Bb}$ \\
\hline EF2 & $1,23 \mathrm{Ab}$ & 2,11Aa & $2,19 \mathrm{Ab}$ & $2,88 \mathrm{Aa}$ \\
\hline
\end{tabular}

(1)Médias seguidas de letras iguais, maiúsculas nas colunas e minúsculas nas linhas, não diferem pelo teste de contraste, a $5 \%$ de probabilidade. EF1,

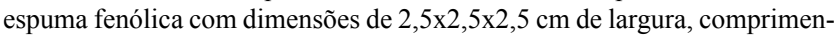
to e altura, respectivamente; e EF2, espuma fenólica com dimensões de $5,0 \times 5,0 \times 3,8 \mathrm{~cm}$ de largura, comprimento e altura, respectivamente.

Pesq. agropec. bras., Brasília, v.50, n.11, p.1027-1032, nov. 2015 DOI: 10.1590/S0100-204X2015001100005 
desenvolvimento de raízes e, consequentemente, a maior absorção de nutrientes pelas plantas, o que pode ter contribuído de maneira positiva para a resposta observada tanto para o acúmulo de massa de matéria seca das plantas quanto para os maiores teores de nutrientes no tecido vegetal (Tabela 3 ).

Houve efeito significativo nos teores de nutrientes na parte área das plantas, ao se comparar os tratamentos contrastantes - EF2/P150 mm x EF1/ P100 mm. Observou-se ainda, nas duas soluções nutritivas, a seguinte sequência decrescente de teores de macronutrientes na parte aérea de copo-de-leite: $\mathrm{K}>\mathrm{N}>\mathrm{Ca}>\mathrm{P}>\mathrm{S}>\mathrm{Mg}$. Em média, os teores de $\mathrm{K}$ na parte aérea das plantas foram cerca de 1,5 vez maior do que os de $\mathrm{N}$, que, por sua vez, foram mais de 5 vezes maiores em relação aos demais macronutrientes. Resultados semelhantes foram obtidos por Carneiro et al. (2015), que também constataram que o $\mathrm{K}$ e o $\mathrm{N}$ foram os nutrientes mais exigidos pelo copo-de-leite, cultivado em substrato à base de fibra de coco.

Em relação aos micronutrientes, obteve-se, de maneira geral, nas duas soluções nutritivas, a seguinte sequência de teores na parte aérea das plantas: $\mathrm{Fe}>\mathrm{Mn}>\mathrm{Zn}>\mathrm{B}>\mathrm{Cu}$.

Tabela 3. Teores de nutrientes na parte aérea de copo-de-leite (Zantedeschia aethiopica) em cultivo hidropônico, com diferentes tamanhos de espuma fenólica e perfis hidropônicos ${ }^{(1)}$.

\begin{tabular}{lcc}
\hline Nutriente & EF1 - Perfil $(100 \mathrm{~mm})$ & $\mathrm{EF} 2-$ Perfil $(150 \mathrm{~mm})$ \\
\hline Macronutriente & & \\
$\mathrm{N}\left(\mathrm{g} \mathrm{kg}^{-1}\right)$ & $23 \mathrm{Bb}$ & $42 \mathrm{Ba}$ \\
$\mathrm{P}\left(\mathrm{g} \mathrm{kg}^{-1}\right)$ & $2 \mathrm{Da}$ & $4 \mathrm{Ca}$ \\
$\mathrm{K}\left(\mathrm{g} \mathrm{kg}^{-1}\right)$ & $40 \mathrm{Ab}$ & $65 \mathrm{Aa}$ \\
$\mathrm{Ca}\left(\mathrm{g} \mathrm{kg}^{-1}\right)$ & $10 \mathrm{Ca}$ & $7 \mathrm{Ca}$ \\
$\mathrm{Mg}\left(\mathrm{g} \mathrm{kg}^{-1}\right)$ & $2 \mathrm{Da}$ & $2 \mathrm{Ca}$ \\
$\mathrm{S}\left(\mathrm{g} \mathrm{kg}^{-1}\right)$ & $2 \mathrm{Da}$ & $3 \mathrm{Ca}$ \\
\hline $\mathrm{Micronutriente}$ & & \\
$\mathrm{B}\left(\mathrm{mg} \mathrm{kg}^{-1}\right)$ & $36 \mathrm{Ca}$ & $31 \mathrm{Ca}$ \\
$\mathrm{Cu}\left(\mathrm{mg} \mathrm{kg}^{-1}\right)$ & $11 \mathrm{Da}$ & $12 \mathrm{Ba}$ \\
$\mathrm{Fe}\left(\mathrm{mg} \mathrm{kg}^{-1}\right)$ & $137 \mathrm{Ab}$ & $143 \mathrm{Aa}$ \\
$\mathrm{Mn}\left(\mathrm{mg} \mathrm{kg}^{-1}\right)$ & $107 \mathrm{Bb}$ & $132 \mathrm{Aa}$ \\
$\mathrm{Zn}\left(\mathrm{mg} \mathrm{kg}^{-1}\right)$ & $99 \mathrm{Bb}$ & $135 \mathrm{Aa}$ \\
\hline
\end{tabular}

(1)Médias seguidas de letras iguais, maiúsculas nas colunas e minúsculas nas linhas, não diferem pelo teste de contraste, a $5 \%$ de probabilidade. $\mathrm{EF} 1$, espuma fenólica com dimensões de $2,5 \times 2,5 \times 2,5 \mathrm{~cm}$ de largura, comprimento e altura, respectivamente; e EF2, espuma fenólica com

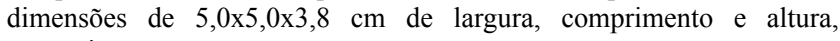
respectivamente.
Assim como observado para o crescimento das plantas (Tabela 2), o uso de EF2/P150 mm resultou em maiores teores de nutrientes na parte aérea das plantas, particularmente de N, K, Fe, Mn e Zn (Tabela 3). Dos nutrientes citados, o $\mathrm{N}$ é o que mais contribui para o crescimento das plantas, pois participa da síntese de proteínas (Malavolta, 2006).

O aumento do conteúdo de água disponível do meio de cultivo melhora a absorção de água e nutrientes (Samartzidis et al., 2005). Isso pode explicar o maior crescimento das plantas e a sua maior absorção de nutrientes, decorrentes do tratamento EF2/P150 mm (Tabelas 2 e 3).

Por meio da determinação dos teores de nutrientes no tecido vegetal, é possível estimar as quantidades de nutrientes absorvidas durante o ciclo das plantas e definir os mais exigidos pelas culturas (Camargo et al., 2005; Guerrero et al., 2012). Com isso, pode-se adequar a adubação para uma nutrição mais equilibrada no cultivo de flores, particularmente do copo-de-leite, em sistema hidropônico, para o qual há escassez de informações.

Em geral, as plantas no tratamento EF2/P150 mm apresentaram maiores taxas fotossintéticas e de transpiração (Figura 1), nas duas soluções utilizadas, o que resultou em maior crescimento (Tabela 3).

As altas taxas fotossintéticas encontradas em EF2/ P150 mm podem ser explicadas pela maior absorção de água e nutrientes nesse tratamento, o que favoreceu a abertura estomática e a absorção de $\mathrm{CO}_{2}$ pelas plantas e aumentou a assimilação do carbono, com efeito positivo nas taxas fotossintéticas, além de ter reduzido os processos de fotorrespiração (Figura 1). A fotossíntese pode ser estimulada pela disponibilidade de $\mathrm{CO}_{2} \mathrm{e}$ luz (Zhou \& Han, 2005). Além disso, a hidroponia estimula o aumento das taxas fotossintéticas em razão das condições ambientais do sistema de cultivo, ao disponibilizar alta concentração de $\mathrm{CO}_{2}$ e temperaturas ideais para a redução da fotorrespiração observada em plantas do tipo $\mathrm{C}_{3}$ (Rosa et al., 2014).

Outro fator considerado é o maior teor de $\mathrm{K}$ na parte aérea do copo-de-leite cultivado em EF2/ P150 mm (65 $\left.\mathrm{g} \mathrm{kg}^{-1}\right)$ quando comparado ao cultivado em EF1/P100 mm (45 $\left.\mathrm{g} \mathrm{kg}^{-1}\right)$ (Tabela 3). Isso pode ter contribuído para a ocorrência de melhores condições para a abertura estomática e trocas gasosas em EF2/P150 mm, evidenciada pelas maiores taxas fotossintéticas e de transpiração, o que permitiu o maior crescimento das plantas nessas condições. 

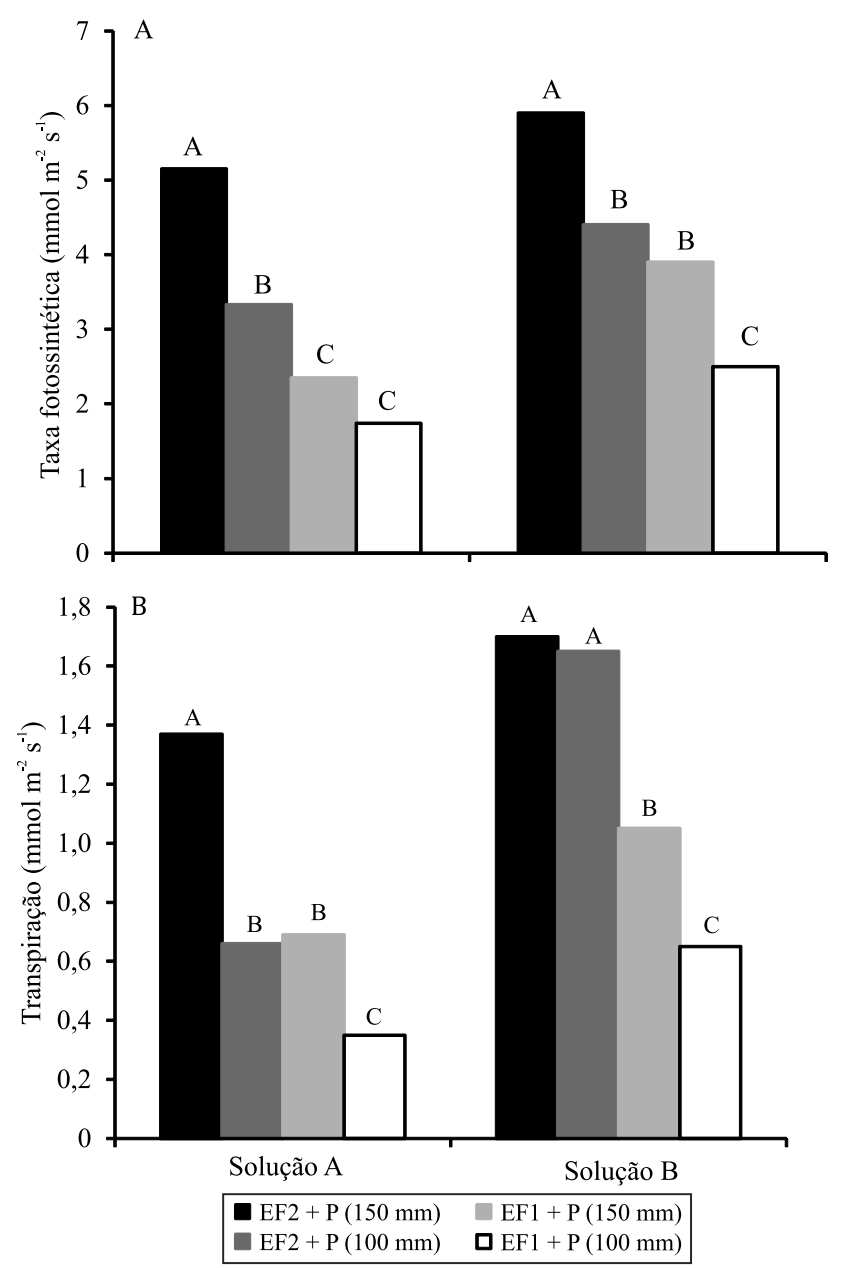

Figura 1. Taxa fotossintética (A) e transpiração (B) em folhas de copo-de-leite (Zantedeschia aethiopica) cultivado em solução nutritiva. EF1, espuma fenólica com

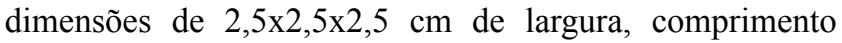
e altura, respectivamente; e EF2, espuma fenólica com

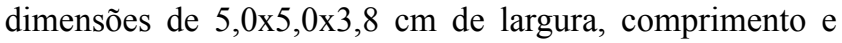
altura, respectivamente. Médias seguidas de letra iguais, maiúsculas, não diferem pelo teste de contraste, a $5 \%$ de probabilidade.

\section{Conclusão}

Plantas de copo-de-leite cultivadas em sistema hidropônico fluxo laminar de nutriente (NFT), com espuma fenólica de 5,0×5,0×3,8 cm e perfil hidropônico de $150 \mathrm{~mm}$, apresentam maior acúmulo de massa de matéria seca na parte aérea e no rizoma, bem como maiores taxas fotossintéticas, independentemente da solução nutritiva.

\section{Agradecimentos}

À Fundação de Amparo à Pesquisa do Estado de Minas Gerais (Fapemig), pelo apoio financeiro.

\section{Referências}

CAMARGO,M.S.de; MELLO, S. da C.;ANTI, G.R.; CARMELLO, Q.A. de C. Crescimento e absorção de nutrientes pelo Aster ericoides cultivado em solo sob estufa. Horticultura Brasileira, v.23, p.271-274, 2005. DOI: 10.1590/S0102-05362005000200021.

CARNEIRO, D.N.M.; COELHO, L.L.; PAIVA, P.D.O.; ALMEIDA, E.F.A.; CARNEIRO, L.F. Evaluation of macronutrient demand in calla lily (Zantedeschia aethiopica). Australian Journal of Crop Science, v.9, p.761-766, 2015.

CHANG, H.S.; CHAKRABARTY, D.; HAHN, E.J.; PAEK, K.Y. Micropropagation of calla lily (Zantedeschia albomaculata) via in vitro shoot tip proliferation. In Vitro Cellular and Developmental Biology-Plant, v.39, p.129-134, 2003. DOI: 10.1079/IVP2002362.

FIGUEIREDO, J.R.M.; ALMEIDA, E.F.A.; FREITAS, G. de F.; REIS, S.N.; LESSA, M.A.; CARVALHO, L.M. de. Desenvolvimento de copo de leite em função da adubação com biofertilizantes. Revista Brasileira de Agropecuária Sustentável, v.4, p.1-5, 2014.

GARCIA-GARZA, J.A.; BLOM, T.J.; BROWN, W.; ROBERTS, D.P.; SCHNEIDER, K.; FREISEN, M.; GOMBERT, D. Increased incidence of Erwinia soft-rot on calla lilies in the presence of phosphorus. European Journal of Plant Pathology, v.110, p.293-298, 2004. DOI: 10.1023/B: EJPP.0000019799.17513.86.

GUERRERO, A.C.; FERNANDES, D.M.; LUDWIG, F. Acúmulo de nutrientes em gérbera de vaso em função de fontes e doses de potássio. Horticultura Brasileira, v.30, p.201-208, 2012. DOI: 10.1590/S0102-05362012000200004.

JUNQUEIRA, A.H.; PEETZ, M. da S. O setor produtivo de flores e plantas ornamentais do Brasil, no período de 2008 a 2013: atualizações, balanços e perspectivas. Revista Brasileira de Horticultura Ornamental, v.20, p.115-120, 2014.

LANDGRAF, P.R.C.; PAIVA, P.D. de O. Exportação de flores e plantas ornamentais do estado de Minas Gerais. Revista Brasileira de Horticultura Ornamental, v.16, p.160-164, 2010.

MALAVOLTA, E. Manual de nutrição mineral de plantas. São Paulo: Agronômica Ceres, 2006. 638p.

MALAVOLTA, E.; VITTI, G.C.; OLIVEIRA, S.A. de. Avaliação do estado nutricional das plantas: princípios e aplicações. 2.ed. Piracicaba: Potafos, 1997. 319p.

POSTALI, G.B.; SILVA, E.C. da; MACIEL, G.M. Produção de híbridos comerciais de tomateiro do grupo cereja cultivados no sistema hidropônico e conduzidos com diferentes números de hastes. Horticultura Brasileira, v.22, p.12-18, 2004.

RIBEIRO, M. de N.O.; PASQUAL, M.; VILLA, F.; CAVALLARI, L. de L. Desenvolvimento in vitro de copo-de-leite: efeito das concentrações de sacarose e de ácido giberélico. Semina: Ciências 
Agrárias, v.30, p.575-580, 2009. DOI: 10.5433/1679-0359.2009v3 0n3p575.

ROSA, A.M.; SEÓ, H.L.S.; VOLPATO, M.B.; FOZ, N.V.; SILVA, T.C. da; OLIVEIRA, J.L.B.; PESCADOR, R.; OGLIARI, J.B. Production and photosynthetic activity of Mimosa verde and Mimosa roxa lettuce in two farming systems. Revista Ceres, v.61, p.494-501, 2014. DOI: 10.1590/0034-737X201461040007.

SAMARTZIDIS, C.; AWADA, T.; MALOUPA, E.; RADOGLOU, K.; CONSTANTINIDOU, H.I.A. Rose productivity and physiological response to different substrates for soil-less culture. Scientia Horticulturae, v.106, p.203-212, 2005. DOI: 10.1016/j. scienta.2005.02.020.
SCHMIDT, D.; SANTOS, O.S.; BONNECARRÈRE, R.A.G.; MARIANI, O.A.; MANFRON, P.A. Desempenho de soluções nutritivas e cultivares de alface em hidroponia.

Horticultura Brasileira, v.19, p.122-126, 2001. DOI: 10.1590/ S0102-05362001000200005.

SILBERBUSH, M.; LIETH, J.H. Nitrate and potassium uptake by greenhouse roses (Rosa hybrida) along successive flower-cut cycles: a model and its calibration. Scientia Horticulturae, v.101, p.127-141, 2004. DOI: 10.1016/j.scienta.2003.10.009.

ZHOU, Y.M.; HAN, S.J. Photosynthetic response and stomatal behavior of Pinus koraiensis during the fourth year of exposure to elevated $\mathrm{CO}_{2}$ concentration. Photosynthetica, v.43, p.445-449, 2005. DOI: 10.1007/s11099-005-0071-5.

Recebido em 27 de abril de 2015 e aprovado em 3 de setembro de 2015 